\title{
A Hybrid Model for Biometric Authentication using Finger Back Knuckle Surface based on Angular Geometric Analysis
}

\author{
K. Usha ${ }^{1}$ \\ ${ }^{1}$ Research Scholar, Department of Computer Science and Engineering, \\ Pondicherry Engineering College, Pondicherry, India \\ ushavaratharajan@gmail.com \\ M.Ezhilarasan ${ }^{2}$ \\ ${ }^{2}$ Associate Professor, Department of Information Technology, \\ Pondicherry Engineering College, Pondicherry, India \\ mrezhil@pec.edu
}

\begin{abstract}
Biometric based personal recognition is an efficient method for identifying a person. Recently, hand based biometric has become popular due to its various advantages such as high verification accuracy and high user acceptability. This paper proposes a hybrid model using an emerging hand based biometric trait known as Finger Back Knuckle Surface. This model is based on angular geometric analysis which is implemented on two different samples of Finger Back Knuckle Surface such as Finger Bend Knuckle Surface and Finger Intact Knuckle Surface for the extraction of knuckle feature information. The obtained feature information from both the surfaces is fused using feature information level fusion technique to authenticate the individuals. Experiments were conducted using newly created database for both Bend Knuckle and Intact Knuckle Surface. The results were promising in terms of accuracy, speed and computational complexity.
\end{abstract}

Index Terms - Finger Back Knuckle Surface, Finger Bend Knuckle Surface, Finger Intact Knuckle Surface, Angular Geometric analysis, Tangents and Secants Method, Feature Information level fusion, Correlation Coefficient

\section{INT RODUCTION}

Biometric Technology is the one which automates the identification of a person based on their physiological and behavioral characteristics for a security system [1]. Among all other biometric traits, hand based biometric modalities are very popular and more advantageous due to their promising results in accuracy, computationally less complex to process and also their user friendliness [2-4]. Many of the works have been done using hand based biometric traits such as palm print, hand geometry, finger Prints, finger geometry, hand vein texture, finger knuckle prints and palm side finger knuckle prints [5-11] etc... In this paper, Finger Back
Knuckle Surface (FBKS) is taken as biometric trait for personal authentication. Here, two different samples of Finger Back Knuckle Surface such as Finger Bend Knuckle Surface and Finger Intact Knuckle Surface are utilized. The internal texture patterns of both the finger back knuckle surfaces are highly unique and a birth feature. The feature information obtained from these patterns is extremely distinctive and able to distinguish between the individuals.

Generally, geometric based biometric systems extract the feature information by exploiting the shape feature of the biometric trait. In many of the existing works on hand biometrics using geometrical analysis extracts the feature information like finger length, finger width, finger area, palm width, palm thickness and palm area etc...[12-13]. The above said feature information will provide limited level of discrimination which leads to difficulty in authenticating the individual. This degrades the performance of the system by increase false acceptance and false rejections. This makes the system to be rarely deployed in real time applications of large users [14]. But these systems have the greatest advantage of high user acceptability and computationally less complex. The above analysis concludes that, there is need for hand based biometric system using geometric analysis which extracts extremely complex information such as angular information to distinguish between the individuals.

This paper solves this issue, by introducing a hybrid model for biometric authentication using Finger Back Knuckle Surface. This hybrid model is composed of angular geometric analysis methods using Tangents and Secants, which is implemented on both the bend knuckle surface and intact knuckle surface to extract the angular information.

This paper is organized as follows. The paper commences with the discussion of existing recognition methods for the finger knuckle prints as a biometric trait in section II. The section III proceeds with the brief discussion about the proposed hybrid model for 
biometric authentication. Section IV and V explores the feature extraction methods for both the Finger Bend Knuckle Surface and Finger Intact Knuckle Surface respectively. In depth analysis of the fusing technique using information score level fusion is done in the subsequent section VI. Illustration of classification technique, which processes the similarities between the reference and input image for biometric authentication, is provided in section VII. Explanations of experimental set up in the mode of verification and identification by conducting two different experiments with the results discussion are presented in the section VIII. This paper concludes with a performance of the proposed hybrid model and with the idea for future work in section IX.

\section{EXISTING WORK}

A number of techniques for personal authentication based on hand based biometric traits such as palm print, finger prints have been proposed in the literature. Most of the hand based biometric trait recognition methods primarily employ three types of feature extraction algorithm, such as, line based, texture based, statistical based [15]. Line based feature extraction schemes are different edge detection methods are used to extract palm lines - principles lines, wrinkles, ridges. The extracted edges directly or being represented other formats. In the line based, palm lines are adopted as biometric feature would result in the possibility for more than one pers on having similar principal lines. [16]

Texture based extraction schemes, in which images are divided into blocks, where the variations exist in either blocks of images are extracted. In these concept techniques like Gabor filters, Ordinal filters, minutiae feature offer promising results. Some popular transform such as Discrete Wavelet transform, Gabor Filters, Discrete Cosine Transforms, Discrete Fourier Transforms are used for extracting features from image data.

In [17] Online palm print identification system employs low-resolution palm print images to achieve effective personal identification. In this system a novel device for online palm print acquisition and an efficient algorithm for fast pal print recognition with 2D Gabor phase encoding scheme is proposed for palm print extraction or representation. In this paper online system refers to a system which captures palm print images using a palm print capture sensor that is directly connected to a system for real time processing. In this authors have identified three issues in developing online palm print identification.1.Palm print acquisition, 2. Palm print feature representation 3. Palm print identification.

In this [18] paper presented a new approach to achieve reliable personal authentication based on simultaneous extraction and combination of multiple biometric features extracted from 3-D and 2-D images of the human hand. The proposed approach in this paper acquires hand images in a contact-free manner to ensure high user friendliness simultaneously captured range and intensity images of the hand are processed for feature extraction and matching. The proposed method of 3-D hand-geometry features explicitly capture curvature variation on the cross-sectional finger segments. Simple and efficient metrics, capable of handling limited variations in the hand pose, are proposed for matching a pair of 3-D hands. In this authors were also introduced a new feature representation, namely, Surface Code, for 3-D palm print which achieves better performance. The experimental results on a database of 177 subjects demonstrate that the 3-D hand-geometry features have high discriminatory information for biometric verification. Besides hand-geometry information, other hand biometric features such as 2-D Palmprint, 3-D Palmprint, and 2-D finger texture can also be simultaneously extracted from the acquired images. Therefore, we investigated the potential of integrating these hand-based features into our unified framework and obtained the best performance when all of the features are combined.

In this [19] paper has presented a new approach for personal authentication using the finger back surface. The developed system automatically extracts the knuckle texture and simultaneously acquires finger geometry features to reliably authenticate the users. The proposed method of knuckle region segmentation, finger ring detection, and the extraction of finger geometry features has been quite effective in achieving higher performance. The system is rigorously experimented on a specially acquired finger back image database from 105 users and achieved promising results. The appearance-based features are extracted from the segmented knuckles using subspace methods and a comparative study is reported. The palm print and fingerprint features can be simultaneously extracted from the palm-side hand images and combined to achieve performance improvement.

In [20] this paper, a new approach to authenticate individuals using triangulation of hand vein images and simultaneous extraction of knuckle shape information. The proposed method of this paper is fully automated and employs palm dorsal hand vein images acquired from low cost, near infrared, contactless imaging. The knuckle tips are used as key points for the image normalization and extraction of region of interest.

In [21] investigates a new approach to quantify the quality of sensed data to generate a reliable estimate on the matching scores. The proposed method is based on the quality of the user templates. In this work, authors have proposed a bimodal biometric system with palm print and hand shape images from a single hand image. The biometric measurement for finger knuckle print is also presented in this work. The user quality is defined as from the associated biometric sample and quantified as a measure of confidence of user biometric sample with its own templates. In this approach a single quality measure for a user is estimated from its genuine training matching scores. This type of quality is the quality for the biometric of the user, rather than the quality of an 
image, and hence, it is termed as user quality. Feature extraction for palm print images is done by extracting effective based on geometrical information or geometry plus interior content. The 17 features are characterized in every hand shape image -Perimeter, four finger lengths, eight finger width, palm width palm length, hand area and hand length are extracted. Matching process is done by means of Euclidean norm. DCT decomposition for the characterization of fixed size palm print images.

The matching scores are generated in stages - 1) Hierarchical matching score from the four topologies of triangulation in the binarized vein structures and 2) From the geometrical features consisting of knuckle point perimeter distances in the acquired images. The weighted score level combination from these two matching scores are used to authenticate the individuals. The weighted score level combination from these two matching scores are used to authenticate the individuals. The experimental result of a system produces high performance with equal error rate of $1.14 \%$.

From the study conducted with the existing works in the literature for recognition methods of finger knuckle print, the analysis shows some of the limitations. In some of the texture analysis methods the accuracy of the system is mainly depends upon the knuckle segmentation methodology. The more accuracy can be obtained with the more accurate segmentation, which gives rise to two different tradeoffs - User acceptability and computational complexity. According to the existing works with the low quality images the accuracy can be obtained by more number of partitioned blocks which in turn increases computational complexity. It has been identified that there is a need of the multimodal biometrics approach which exploits the subset of features by means of geometrical analysis, which can be computationally less complex.

\section{THE HYBRID MODEL}

Fig. 1 has shown the hybrid implementation model for biometric authentication. In this, Finger Back Knuckle Surface is captured in two different samples 1) Finger Bend Knuckle Image 2) Finger Intact Knuckle Image. The captured images are preprocessed to extract the region of interest from the image for further processing. The biometric authentication system have two stages, the first stage is an 'enlistment' stage or registration stage - In which bio metrics modal Finger Back Knuckle Surface is mobilized. The measurement of biometric trait is done and the information of the biometric characteristic measurements is enrolled in the database. The second stage is 'liberate' stage or Input stage - In which mobilization of a new input biometric characteristic is done and then authentication process. In this, the enlistment stage and liberate stage is done by means of four components, they are 1) Feature point identification component 2) Feature information extraction and representation component 3) Feature information level fusion component and finally
4)Classification component for matching process. The decision is made according to the output of matching process. The proposed system have two fold advantages, 1) This hybrid model biometric system overcomes the limitations of unimodal, such as degradation in accuracy for large user application and easy to encroach the trait etc... 2) Feature extraction based on geometric analysis method, which is done by concentrating in the less number of features, in turn reduces computational complexity.

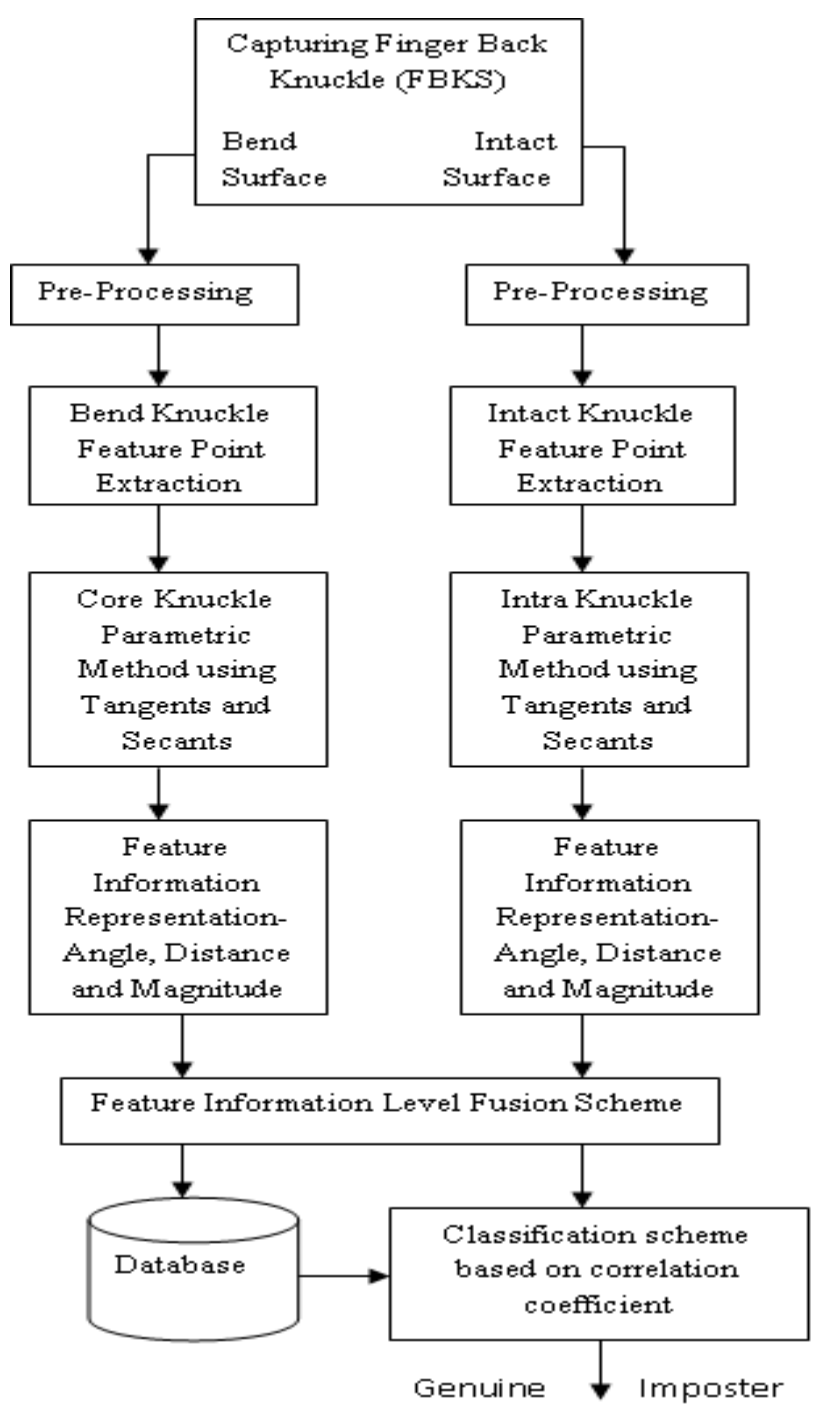

Figure 1: The Hybrid Implementation Model for Biometric Authentication

\section{FINGER BEND KNUCKLE SURFACE} PREPROCESSING AND FEATURE EXTRACTION METHOD

\section{A. PRE - PROCESSING AND ROI EXTRACTION}

The preprocessing of the captured Finger Bend Knuckle Surface image is done to extract the knuckle patterns. Fig. 2 illustrates the pre processing of finger bend knuckle surface. The segmentation of the captured image is done by incorporating the coordinate system. This is achieved by defining the $x$ - axis and y-axis of the 
captured image. The base line of the finger is taken as $x$ axis. The $y$-axis for bend surface knuckle patterns in the finger is defined by means of convex curves determined from the edge record of the canny edge detection algorithm [18]. The curvature convexit ies of the obtained convex curves were determined for bend knuckle pattern. The Y axis of the knuckle is resolute by means of the curvature complexity which is nearly equal to zero at the center point.

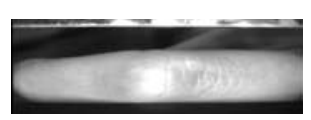

A
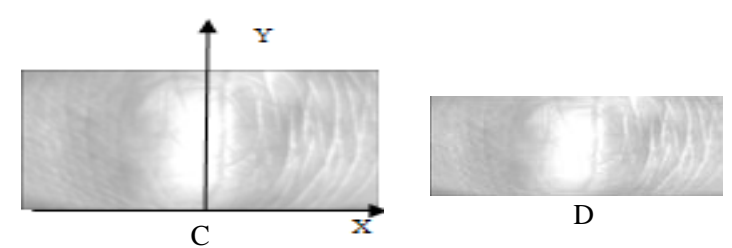

Figure 2: A) Finger Bend Surface B) Convex Curves of Bend Surface C) Co-ordinate system for Finger Bend Knuckle Surface D) ROI of Finger Bend Knuckle Surface.

By this method, ROI is extracted from the FBKS which of $100 \times 80$ pixel size for bend surface knuckle region. The extracted ROI image consists of area contiguous to the joints of the FBKS, which consists of points, lines and curves for feature information extraction.

\section{B. FINGER BEND SURFACE FEATURE POINT EXTRACTION METHOD.}

Finger bend knuckle surface image shown in the fig.2(a) are subjected to normalization and segmentation to obtain ROI. Knuckle feature points were identified in this experiment are knuckle edge points and Knuckle mid points. Canny edge detector algorithm [22] is used to identify the curved feature from both the sides of finger back knuckle surface. These curves can be called as hybrid convex curves, since it curves both leftwards and rightwards as shown in the fig. 2 (b). From the identified convex curves, knuckle edge points were calculated. It is done by establishing sequence of points that the line passes through. Edge in the obtained is identified by the concept that the edges are the areas with the contrast in intensity. The intensity level in the edge varies from one pixel to the next pixel. Based on this criterion, the canny algorithm is the gradient based algorithm which highlights the maximum derivative region by restraining the pixel that is not maximum. Edge thresholding is used in this algorithm to identify the edge pixel which is above the high threshold. The edge points identified by means of convex curves can be named as Finger Bend Knuckle Edge Right (FBKER) and Finger Bend Knuckle Edge Left (FBKEL).

\section{Knuckle Mid Identification}

Knuckle midpoint identification is done by the determination of $\mathrm{X}$-axis and Y-axis of the knuckle image as shown in the fig. 2 (c). The axis identification is also done based on the canny edge detection algorith $m$. This can be captured from the acquired image itself. By considering the bottom boundary is a straight line, local coordinate system is determined. The hybrid curves identified from the canny edge algorithm curves both the sides. There are some area around the middle surface of the finger back knuckle surface contains some of the lines whose curvature convexity is nearly equal to zero. One such line is identified around the center of the curved surface of the knuckle and it is used to set the Yaxis. The midpoint of the $\mathrm{Y}$ axis line is taken as Finger Bend Knuckle Mid Point (FBKMP).

\section{CORE KNUCKLE PARAMETRIC METHOD}

Core Knuckle Parametric Method is a Geometrical Analysis Method (KGAM), which enables to construct various geometrical structures on identified knuckle features so that the feature information about the bend surface knuckle can be easily extracted. In this method, geometrical structures like circles, Tangents, Secants were constructed using the identified knuckle contours such as Finger Bend Knuckle Edge Right (FBKER), Finger Bend Knuckle Edge Left (FBKEL) and Finger Bend Knuckle Mid Point (FBKMP). Concentric circles were constructed on knuckle feature with the distance between FBKER and FBKMP as radius and the distance between FBKEL and FBKMP as another circle radius.

In each circle the construction of tangent using knuckle edge points which is away from the of both sides of knuckle surface. Tangent is constructed by drawing a straight line joining both the edge points and Mid Point of the Finger Bend Knuckle Surface. Construct a bisector for the line and the distance between the FBKMP and the bisector line is taken as the magnitude to locate the points $\mathrm{B}$ and $\mathrm{C}$ on the either side of the circumference of the circle from the bisector line. These are the points at which two tangents touches on either side of the circumference circle as shown in the fig. 3. The angle formed by the two tangents can be called as Convex Right Angle Tangent Tangent (CRATT) and Convex Left Angle Tangent Tangent (CLATT)

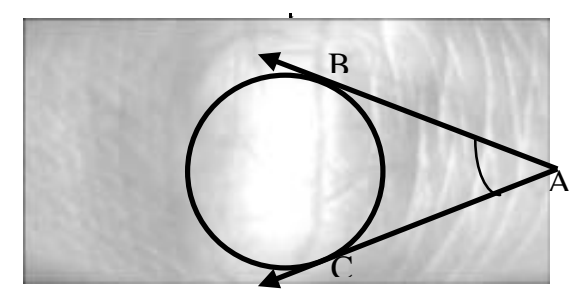

Figure 3: Construction of Tangents on the either of the circles.

Two Secants were drawn as shown in the fig. 4 on either side of the circle by satisfying the following condition specified in (1).

AB. $A D=A C \cdot A E$ 


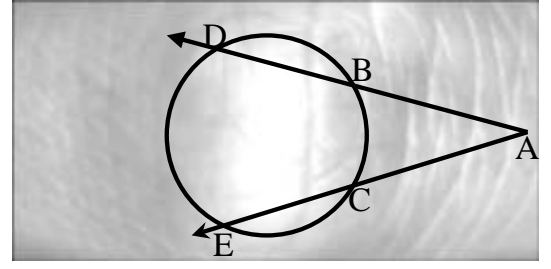

Figure 4: Construction of Secants on the either of the circles

Angle formed by the two secants on either side of the circle can be called as Convex Right Angle Secant Secant (CRASS) and Convex Left Angle Secant Secant (CLASS).

A tangent and a secant were drawn as shown in fig. 5 on either side of the circle by satisfying the following condition specified on (2).

$$
\text { AB. } \mathrm{AD}=\mathrm{AC}^{2}
$$

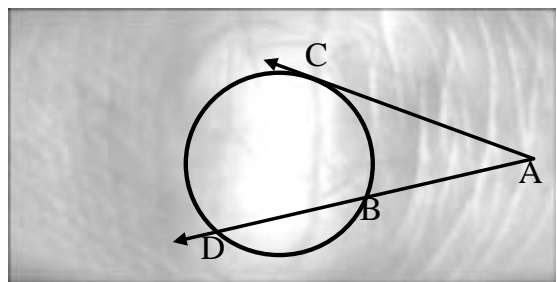

Figure 5: Construction of Tangents and Secants on the either of the circles

Angle formed by a tangent and secant on either side of circle can be called as Convex Right Angle Tangent and Secant (CRATS) and Convex Left Angle Tangent and Secant (CLATS).

Angle formed by the Tangent-Tangent, TangentSecant and Secant-Secant are calculated by (3) and (4)

$\angle C L A T T=\angle C L A S S=\angle C L A T S=\frac{\text { far } \operatorname{arc}(\text { length })-\text { near } \operatorname{arc}(\text { length })}{2}$

$\angle C R A T T=\angle C R A S S=\angle C R A T S=\frac{\text { far } \operatorname{arc}(\text { length })-\text { near } \operatorname{arc}(\text { length })}{2}$

The far arc and near arc and near arc length can be calculated by computing the central angle formed by the arc sector, which can be given by the following (5),(6),(7) and (8)

$$
\begin{aligned}
& \angle \text { NearArc }(\text { angle })=\frac{\mathrm{a}^{2}+\mathrm{b}^{2}+\mathrm{c}^{2}}{2 \mathrm{bc}} \\
& \text { NearArc (length) }=\frac{\text { NearArc }(\text { angle })}{180} * \pi * \gamma \\
& \angle \text { FarArc }(\text { angle })=360-\angle \text { NearArc }(\text { angle }) \\
& \text { FarArc (length })=\frac{\text { FarArc }(\text { angle })}{180} * \pi * \gamma
\end{aligned}
$$

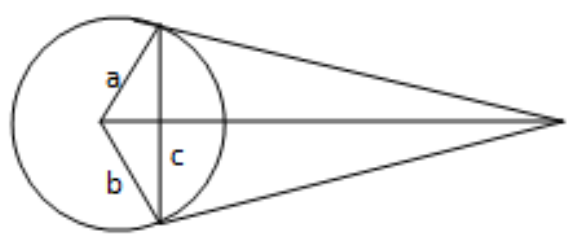

Figure 6: Representation of Arc length in Finger Bend Knuckle Surface.

Six different angle measurements were done for single biometric knuckle image and stored in a two vectors named as $V_{\text {ref right }}$ and $V_{\text {ref left }}$ for both left side and right arc angles respectively.

\section{FINGER INT ACT KNUCKLE SURFACE}

\section{PREPROCESSING AND FEATURE EXTRACTION METHOD}

\section{A. PRE - PROCESSING AND ROI EXTRACTION}

The preprocessing of the captured Finger Intact Knuckle Surface image is done to extract two different knuckle patterns. Fig. 7 illustrates the two different knuckle pattern namely Primary knuckle and Core Knuckle of the Finger Intact Knuckle surface. The segmentation of the captured Finger Intact Knuckle image is done by incorporating the coordinate system. This is achieved by defining the $x$ - axis and $y$-axis of the captured image. The base line of the finger is taken as $\mathrm{x}$ axis. The y-axis for two different knuckle patterns in the finger is defined by means of convex curves determined from the edge record of the canny edge detection algorithm [18]. The curvature convexities of the obtained convex curves were determined for both primary and core knuckle Pattern. The Y axis of the primary and core Knuckle are resolute by means of the curvature complexity which is nearly equal to zero at the center point. By this method, ROI is extracted from the FBKS which of $100 x 80$ pixel size for primary knuckle region and 220x110 pixel size for core knuckle region. The extracted ROI image consists of area contiguous to the joints of the FBKS, which consists of points, lines and cuvees for feature information extraction.

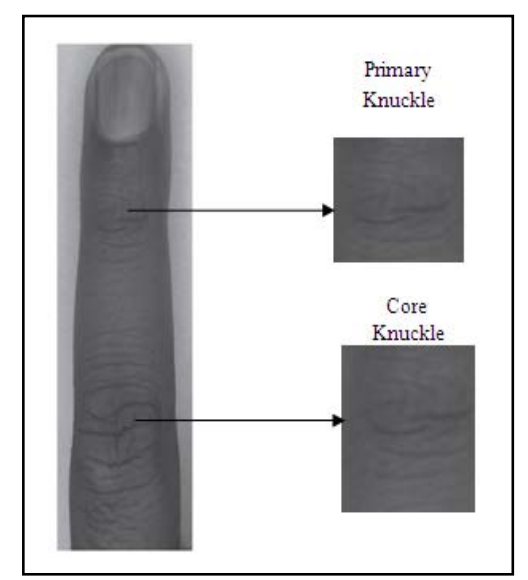

Figure 7: Illustration of Primary and Core Knuckle ROI pattern of Finger Intact Knuckle Surface 


\section{B. FINGER INTACT SURFACE FEATURE POINT EXTRACTION METHOD}

This method incorporates the features of both the core knuckle and primary knuckle. This method initially identifies FBKS feature points such as knuckle edge points and knuckle mid points from both the primary and core knuckle surfaces.

\section{Knuckle Edge Identification}

Knuckle edge points were identified by means of Canny edge detector algorithm [13][17], which identifies the curved feature from both primary knuckle and core knuckle surfaces of FBKS. From the identified convex curves, knuckle edge points were calculated. It is done by establishing sequence of points that the line passes through. Edge in the obtained is identified by the concept that the edges are the areas with the contrast in intensity. The intensity level in the edge varies from one pixel to the next pixel. Based on this criterion, the canny algorithm is the gradient based algorithm which highlights the maximum derivative region by restraining the pixel that is not maximum. Edge thresholding is used in this algorithm to identify the edge pixel which is above the high threshold. The edge points identified by means of convex curves can be named as Primary Knuckle Edge Right (PKER) and Primary Knuckle Edge Left (PKEL). For the core knuckle edge points are named as Core Knuckle Edge Right (CKER) and Core Knuckle Edge Left (CKEL).

\section{Knuckle Mid Point Identification}

Knuckle mid identification is done by the determination of $\mathrm{X}$-axis and $\mathrm{Y}$-axis of the knuckle image as shown in the fig. 2 (d). The axis identification is also done based on the canny edge detection algorith $\mathrm{m}$. The $\mathrm{X}$-axis can be captured from the acquired image itself. By considering the bottom boundary is a straight line, local coordinate system is determined. The hybrid curves identified from the canny edge algorithm curves both the sides. There are some area around the middle surface of the FBKS contains some of the lines whose curvature convexity is nearly equal to zero. One such line is identified around the center of the curved surface of the knuckle and it is used to set the Y-axis. The midpoint of the $\mathrm{Y}$ axis line is taken as Primary Knuckle Mid Point (PKMP). Similarly, Y' is y-axis of the core knuckle, the knuckle mid point is identified as the midpoint of the $\mathrm{Y}^{\prime}$ axis and it is named as Core Knuckle Mid Point (CKMP).

\section{INTRA KNUCKLE PARAMETRIC METHOD}

Intra Knuckle Parametric method using Tangents and Secants of proposed work enables to construct various geometrical structures on identified knuckle features. In this method, geometrical structures like circles, Tangents, Secants were constructed using Primary knuckle edge points, Core knuckle edge points, Primary knuckle mid point and Core knuckle mid point. From the identified Core Knuckle Mid Point (CKMP), a circle is constructed with the distance between CKMP and CKER as radius and it is named as circle ' $R_{c}$ '. From the identified Primary Knuckle Mid Point (PKMP), which is away from the circle, the following structures were constructed, (1) Two tangents were constructed to the circle, (2) Two Secants were constructed to the circle, (3) A tangent and a secant is constructed to the circle. All the structures were constructed from the identified knuckle feature points are shown in the fig. 8.

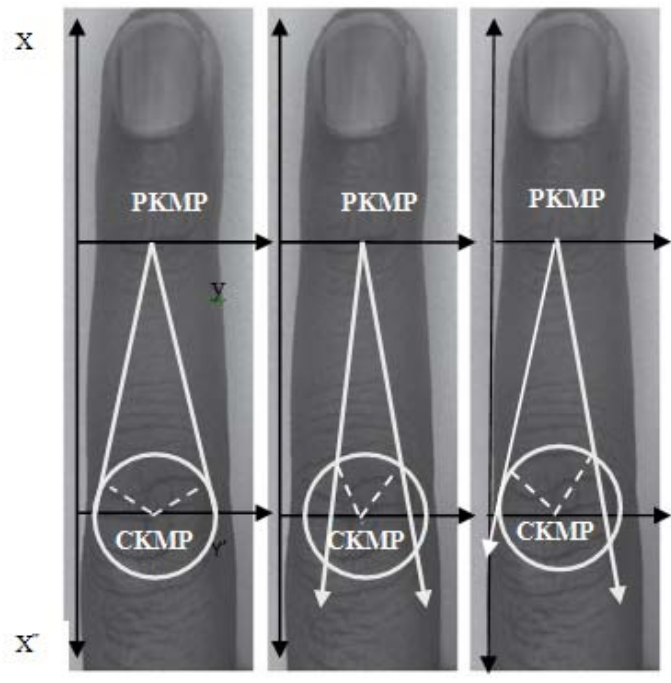

Figure 8: Illustration of Tangent and Secant Method using Core Knuckle Parameters

Similar structures were constructed using the feature points of primary knuckle. From the identified Primary Knuckle Mid Point (PKMP), a circle is constructed with the distance between PKMP and PKER as radius and it is named as $R_{p}$. From the identified Core Knuckle Mid Point (PKMP), which is away from the circle $R_{P}$, the following structures were constructed, (1) Two tangents were constructed to the circle, (2) Two Secants were constructed to the circle, (3) A tangent and a secant is constructed to the circle. All these structures were constructed from the identified knuckle feature points are shown in the fig. 9 .

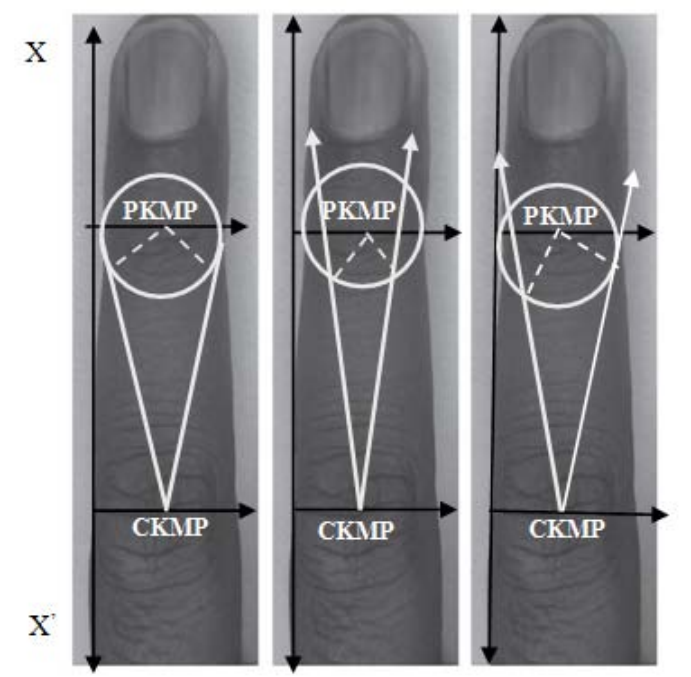

Figure 9: Illustration of Tangent and Secant Method using Primary Knuckle Parameters 
Let $\theta$ be the angle formed by each set of constructed geometrical structures such as (1) Tangent-Tangent (2) Secant - Secant (3) Tangent - Secant. The angle value of the $\theta$ can be calculated by the formu la given in (9).

$$
\theta=\frac{\text { far arc length }- \text { near arc length }}{2}
$$

The far arc can be defined as the arc formed by the each set of constructed geometrical structure which is away from the point from where these structures were constructed.

The near arc can be defined as the arc formed by each set of constructed geometrical structure which is nearer for the point from which these structures were constructed. The far arc and near arc lengths can be computed by calculating the angle formed by the sector which is equivalent to the arc length. The far and near arc angle can be calculated by the above said equations.

Angular information extracted by means of tangents and secants method by considering both the primary and core knuckle parameters are represented in the form of vectors. The obtained angular information can be named as Core Angle Tangent-Tangent (CATT), Core Angle Secant-Secant (CASS), Core Angle Tangent-Secant (CATS), Primary Angle Tangent-Tangent (PATT), Primary Angle Secant-Secant (PASS) and Primary Angle Tangent-Secant (PATS). All the angular information was stored in two different vectors known

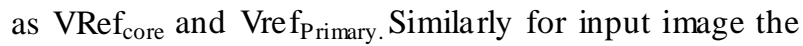
vectors obtained are VInp core $_{\text {and }}$ VInp $p_{\text {Primary }}$

\section{FEATURE INFORMATION LEVEL FUSION}

The Feature sets extracted from the multiple modalities can be fused together to define a new feature vector for the individual for identification can be called as feature information level fusion. Here, Feature information obtained from both the Finger Bend Knuckle Surface and Finger Intact Knuckle Surface using Angular Geometric Analysis Method are fused as single vector information for personal recognition. Both the feature information is fused by combining serially the two sets of feature vector into single-Vector. Suppose $\alpha$ be the information vector 1 with n dimension and $\beta$ being the information vector 2 with m dimension obtained from the arbitrary information $\xi$. The combination of these vector can be done by using weighted amalgamation method. This method gives weights are fixed according to the dimensionality of the vector. $\psi=\left(\begin{array}{c}\alpha \\ \Phi \beta\end{array}\right)$, where $\boldsymbol{\Phi}$ is the weight, can be called as amalgamation coefficient. This amalgamation coefficient is obtained by giving higher order weights to higher dimensional vector, since higher dimensional vector provides more powerful information than the low dimensional vector. This is achieved by comparing the dimensions $\mathrm{n}$ and $\mathrm{m}$, and the value of $\Phi$ is obtained fro $m$ values between $\omega$ and $\omega^{2}$, where $\omega=n / m$, if $n>m$.

\section{CLASSIFICATION SCHEME}

The Classification Scheme is based on Correlation Coefficient Analysis. The Correlation Coefficient is a number that summarizes the direction and closeness of linear relations between two values. This analysis is made between reference vector information and input vector information.

Let $\mathrm{x}$ be the value from reference vector $\mathrm{V}_{\text {ref }} \mathrm{Y}$ be the value from input vector $V_{\text {inp }}$

The coefficient can be calculated in (10)

$$
\rho=\frac{\sigma_{x y}}{\sigma_{x} \sigma_{y}}
$$

Value is close to 1 ; indicate the similarity measure, when the value converges to 0 , dissimilarity between values.

\section{EXPERIMENTAL RESULTS AND DISCUSSION}

\section{A. DATA SET FOR EXPERIMENT}

Date set for FBKBS images were captured using automated low cost contact less method using low resolution camera and in peg free environment. In this FBKBS images were collected from 165 volunteers, including 125 males and 40 females. A mong them, 143 subjects were 20-30 years old and the others were 30 50 years old. These samples were collected in two separate sessions. In each session, the subject was asked to provide 6 images for each of the left index finger, the left middle finger, the right index finger, and the right middle finger. Therefore, 48 images from 4 fingers were collected from each subject. In total, the database contains 7,920 images from 660 different fingers. The average time interval between the first and the second sessions was about 25 days. The maximum and minimum intervals were 96 days and 14 days, respectively.

Data set for FBKIS images were captured using automated low cost contact less method using low resolution camera and in peg free environment. The entire back surface of four different fingers were captured from 120 volunteers, including 60 males and 60 females. The samples were collected in a two different sessions. In each session subjects were asked to give five images of each of the left index finger, Right index finger, Left middle finger and Right middle finger. There are totally 40 images for a subject. In total, the database contains 4800 images from 480 different fingers.

\section{B. EXPERIMENTAL RESULTS}

The results of the experimental analysis conducted both in verification and identification mode is illustrated 
as follows: In these results Genuine Acceptance Rate (GAR) obtained for the five different values of False Acceptance Rate (FAR). Two different methods Angular Geometric Analysis Method using Bend Knuckle Surface and Intact Knuckle Surface were implemented. Initially results were obtained for single sample of the FBKS modal, such as, left index FBKS, left middle for FBKS, right index FBKS, right middle FBKS separately by applying both the methods. Secondly, results were obtained by fusing feature information obtained from the four different fingers using feature information level fusion method. Finally, results were obtained by combining the feature information obtained by the two different modalities of FBKS and obtained result is compared with the existing methods. Three different existing geometrical analysis and texture analysis method are taken for comparison. The description of existing method which is taken for comparison is illustrated below:

Finger Knuckle Geometric Method: In this method, feature extraction of the finger knuckle print is obtained by means of both geometrical analysis and texture analysis. The knuckle texture information identified by means of PCA, ICA and LDA algorithms. The result obtained for the fixed dimension of feature vector.

Palm and Knuckle Print Texture Method: In this method, palm print texture information is obtained by applying PCA algorithm to the entire image. The hand shape information is obtained by various control points in the acquired hand image. In addition to that, finger knuckle information is used to improve the performance.

Hand Vein Geometric Method: In this method, Delaunay triangulation is used to extract hand vein structure information and geometrical methods for extracting the knuckle shape information. Using this method, this limits to selected set of feature vector. The following table I illustrates the comparison of Intra knuckle parametric method with the existing work.

TABLE I: COMPARISON OF THE PROPOSED HYBRID MODEL WITH THE E XISTING METHODOLOGY

\begin{tabular}{|c|l|c|}
\hline REFERENCE & DATA SET & EER \% \\
\hline $\begin{array}{c}\text { Finger Knuckle } \\
\text { Geometric Method }\end{array}$ & 103 samples of FKP & 1.39 \\
\hline $\begin{array}{c}\text { Palm and Knuckle } \\
\text { Texture Method }\end{array}$ & $\begin{array}{l}\text { 100 samples of Hand } \\
\text { Image }\end{array}$ & 2.20 \\
\hline $\begin{array}{c}\text { Hand Vein } \\
\text { Triangulation Method }\end{array}$ & $\begin{array}{l}\text { 100 Samples of Hand } \\
\text { Back Surface }\end{array}$ & 1.77 \\
\hline $\begin{array}{c}\text { Hybrid Model using } \\
\text { FBKS }\end{array}$ & $\begin{array}{l}\text { 7920 images of } \\
\text { FingerBend Knuckle. } \\
\text { 4800 images of } \\
\text { FingerIntact Knuckle. }\end{array}$ & 0.87 \\
\hline
\end{tabular}

The following graphical illustrations are the results obtained by implementing the hybrid Model using Finger Back Knuckle Surface. Fig. 10 and 11 illustrates the Receiver operational Characteristics obtained by implementing the core knuckle and intra knuckle parametric method in Finger Bend and Finger Intact Knuckle Surface image respectively.

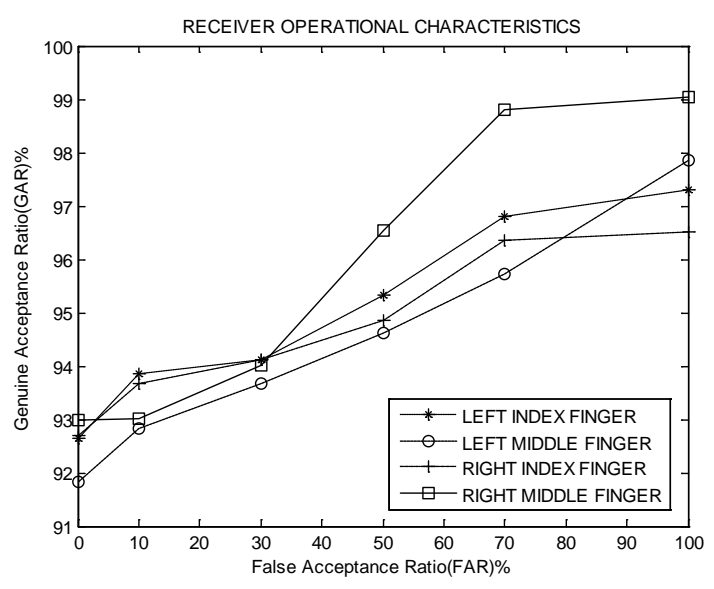

Figure. 10: ROC obtained from the individual using Core Knuckle Parametric Method based on Tangents and Secants Method.

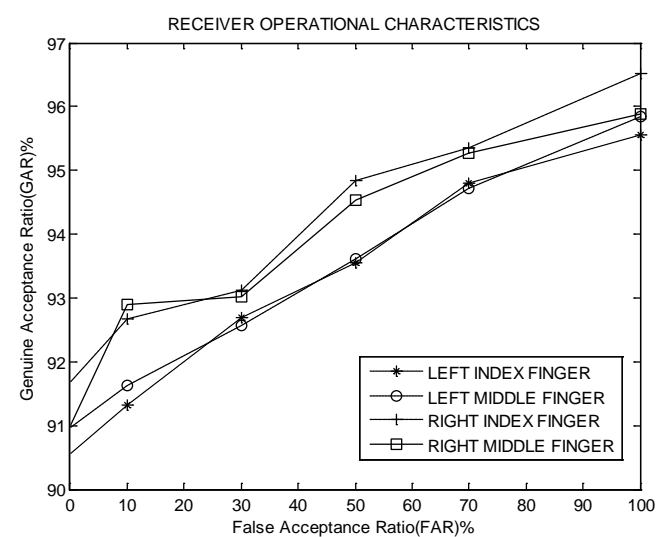

Figure 11: ROC obtained from the individual using Intra Knuckle Parametric Method based on Tangents and Secants Method.

Fig. 12 illustrates the receiver operational characteristics obtained by implementing the core knuckle and Intra Knuckle parametric method in Finger Bend and Finger Intact Knuckle Surface image respectively. Score values are generated by fusing the feature information from all the four fingers.

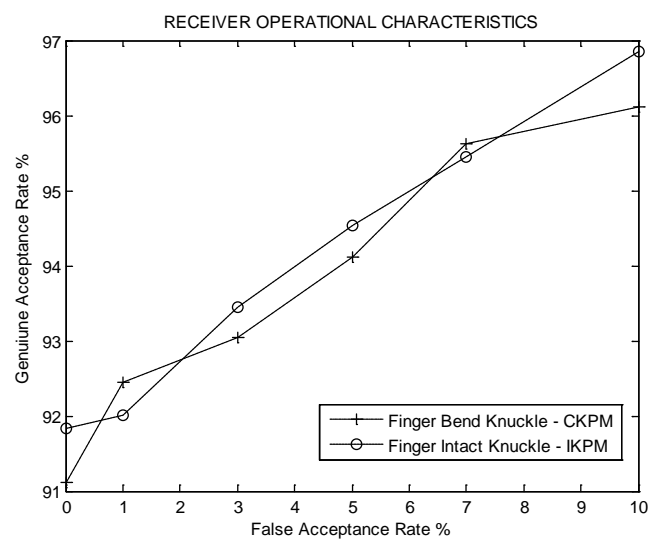

Figure. 12: ROC obtained from the combined performance of all the four finger of the both core knuckle and intra knuckle parametric method 
Fig.13 illustrates the Receiver operational Characteristics obtained by implementing the core knuckle and Intra Knuckle parametric method in Finger Bend and Finger Intact Knuckle Surface image respectively.

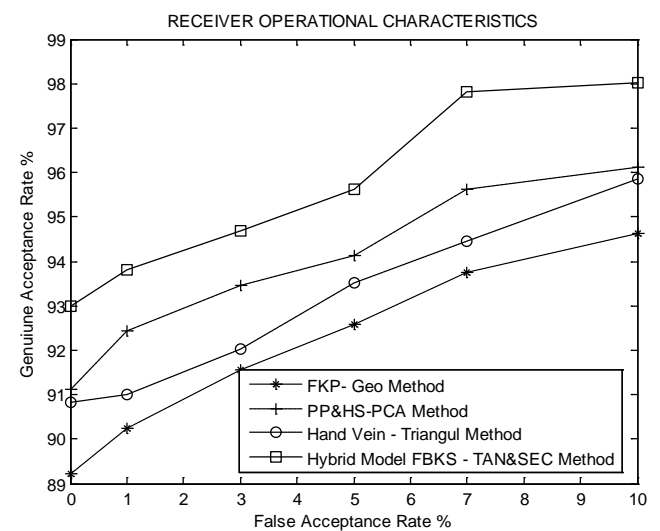

Figure. 13: ROC obtained from the Hybrid Model based on Tangents and Secants Method and compared with the existing Methods

Score values are generated by fusing the feature information from all the four fingers. The proposed Hybrid Model is compared with the existing hand based biometric system.

\section{RESULTS DISCUSSION}

The proposed Hybrid Model for personal recognition using Finger back knuckle surface achieves better performance than the existing techniques in the literature.

The discussions about the results of an experimental study were presented below.

(i) Graphical illustrations suggest that there is significant improvement in performance using the proposed method.

(ii) In the conducted experiments, there is no single fusion combination best performing type. Different fusion combination performs better during the different experimental types.

(iii) From this experiment, It is also been proved that the Finger back knuckle surface as a biometric identifier achieves better performance similar to that of other biometric traits which has been under research for longer time such as finger prints.

(iv) When using the finger knuckle prints and finger prints along with palm prints in the Verification mode experiment there was $1.68 \%$ difference in performance.

(v) Also using the finger knuckle print and finger prints fused with palm prints in the Identification mode experiment, there was $1.51 \%$ difference in the performance obtained.

\section{CONCLUSION}

This paper presented a new hybrid model to achieve effective performance in personal authentication system based on Intra modal biometric which utilizes Finger Back Knuckle Surface as a Biometric Trait. This approach acquires the knuckle images in a contact free manner to enhance user friendliness. In order to extract unique information from the FBKS, we introduce two different representations, namely Finger Bend Knuckle Surface and Finger Intact Knuckle surface. The hybrid model methods were based on geometric analysis. Tangents and Secants method on both finger bend and Finger intact knuckle surface explicitly captures the angular feature information by constructing the geometrical structures like circles, Tangents and Secants etc... The potentiality of the innovated hybrid model was investigated by means of experiments. The quantification of performance improvement is done based on accuracy, and other based on computation complexity.

\section{REFERENCES}

[1] Bolle R.M Cornell j.h, PANKANTI S. Ranjith, N.K SENIOR A.W Guide to Biometrics 2003, Network Springer Verlag.

[2] A jay Kumar, and Ch. Ravikanth "Personal Authentication Using Finger Knuckle Surface", IEEE TRANSACTIONS ON INFORMATION FORENSICS AND SECURITY, VOL. 4, NO. 1, MARCH 2009.

[3] Y. Gao, S.C. Hui, and A.C.M. Fong, “A MultiView Facial Analysis Technique for Identity Authentication,” IEEE Pervasive Computing, vol. 2, no. 1, 2003, pp. 38-45.

[4] Maylor K.H. Leung, A.C.M. Fong, and Siu Cheung Hui Palmprint Verification for Controlling Access to Shared Computing Resources Published by the IEEE Computer Society 2007 IEEE.

[5] Goh Kah Ong Michael and Tee Connie, Andrew Teoh Beng Jin Robust Palm Print and Knuckle Print Recognition System Using a Contactless Approach, 2010 IEEE.

[6] Abdallah Meraoumia1, Salim Chitroub1 and Ahmed Bouridane2, Fusion of Finger-Knuckle-Print and Palmp rint for an Efficient Multi-biometric System of Person Recognition, 2011 IEEE.

[7] Ajay Kumar and David Zhang,” Personal Recognition Using Hand Shape and Texture”, IEEE TRANSACTIONS ON IMAGE PROCESSING, VOL. 15, NO. 8, AUGUST 2006.

[8] J. Fierrez-Aguilar, J. Ortega-Garcia, J. GonzalezRodriguez, and J. Bigun, "Discriminative multimodal biometric authentication based on quality measures,” Pattern Recognit., vol. 38, no. 5, pp. 777-779, 2005.

[9] D. E. Maurer and J. P. Baker, "Fusing multimodal biometrics with quality estimates via a Bayesian 
belief network,” Pattern Recognit., vol. 41, no. 3, pp. 821-832, 2007.

[10] A. Kumar and D. Zhang, "Personal recognition using hand shape and texture,” IEEE Trans. Image Process., vol. 15, no. 8, pp. 2454-2461, Aug. 2006.

[11] S. Ribaric and I. Fratric, “A biometric identification system based on eigenpalm and eigenfinger features,” IEEE Trans. Pattern Anal. Mach. Intell., vol. 27, no. 11, pp. 1698-1709, Nov. 2005.

[12] A. Kumar and D. Zhang, "Hand geometry recognition using entropy based discretization," IEEE Trans. Inf. Forensics Security, vol. 2, no. 2, pp. 181-187, Jun. 2007.

[13] S. Malassiotis, N. Aifanti, and M. G. Strintzis, "Personal authentication using 3-D finger geometry," IEEE Trans. Inf. Forensics Security, vol.1, no. 1, pp. 12-21, Mar. 2006.

[14] Q. Li, Z. Qiu, D. Sun, J. Wu, "Personal Identification using knuckleprint," in SINOBIOMETRICS, Guangzhou, 2004, pp. 680689.

[15] A.Kong, D.Zhang, and M.Kamel, "survey of palmprint recognition”, Palm print recognition, Vol.42, pp. 1408-1418,2009.

[16] Hafiz Imtiaz and Shaikh Anowarul Fattah,” A DCT-based Feature Extraction Algorithm for Palmprint Recognition”, 2010 IEEE.

[17] David Zhang, Senior Member, IEEE, Wai-Kin Kong, Member, IEEE, Jane You, Member, IEEE, and Michael Wong "Online Palmprint Identification”, IEEE TRANSACTIONS ON PATTERN ANALYSIS AND MACHINE INTELLIGENCE, VOL. 25, NO. 9, SEPTEMBER 2003.

[18] Vivek Kanhangad, Ajay Kumar, Senior Member, IEEE, and David Zhang, Fellow, IEEE “A Unified Framework for Contactless Hand Verification”, IEEE TRANSACTIONS ON IMAGE PROCESSING, VOL. 15, NO. 8, AUGUST 2006.

[19] Ajay Kumar, Senior Member, IEEE, and Ch. Ravikanth "Personal Authentication Using Finger Knuckle Surface” IEEE TRANSACTIONS ON INFORMATION FORENSICS AND SECURITY, VOL. 4, NO. 1, MARCH 2009.

[20] Ajay Kumar and K.Venkata prathyusha, "Personal authentication using Hand vein Triangulation and Knuckle shape”, IEEE Transactions on Image processing, VOL 18, No.9, September 2009.

[21] Ajay Kumar and David Zhang, "Improving Biometric Authentication Performance from the User Quality”, IEEE Transactions on Instrumentation and Measurement. Vol.59, No.3 March 2010.

[22] Paul Bao,Lei Zhang, and Xiaolin Wu.: Canny Edge Detection Enhancement by Scale Multiplication.: IEEE Transactions On Pattern Analysis And Machine Intelligence, Vol. 27, no. 9,(2005).

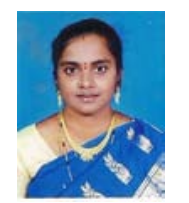

K.USHA is a Research Scholar. She is currently working toward the Ph.D. degree in the Computer Science and Engineering majoring in Biometric Technology in the Pondicherry Engineering College, Pondicherry, INDIA. Her research interests include Hand based Biometrics, digital image processing and Software Reliability.

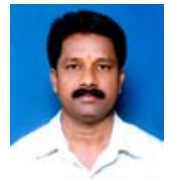

Dr. M.Ezhilarasan was born on May 30, 1968. He has obtained Bachelor of Technology in Computer Science and Engineering, Master of Technology in Computer Science and Engineering and Ph. D. in Computer Science and Engineering from Pondicherry University in 1990, 1996 and 2007 respectively. He is an Associate Professor and Head of Information Technology, Pondicherry Engineering College, Puducherry, India. He is life member of Indian Society for Technical Education (ISTE). He has published 45 papers in National and International Conference Proceedings and Journals. His research interests include Multimedia Data Compression and Multi-Bio metrics. 